\title{
Adherence to Supervised and Unsupervised Home Exercise Program on Motor Milestones in Spastic Cerebral Palsy
}

\author{
Namrata Patil*, G Varadhrajulu and Mandar Malawade \\ Department of Pediatric Physiotherapy, Krishna College of Physiotherpay, India
}

Submission: February 09, 2018; Published: March 19, 2018

*Corresponding author: Namrata Patil, Department of Pediatric Physiotherapy, Krishna College of Physiotherpay, KIMS, Karad 415110, India, Email: dr.namratakcpt@gamil.com

\begin{abstract}
Background: Cerebral palsy (CP) describes a group of developmental disorders of movement and posture, causing activity restriction or disability that is attributed to disturbances occurring in the fetal or infant brain. The motor impairments may be accompanied by a seizure disorder and by impairment of sensation, cognition, communication and behavior. The hallmark sign of group of disorders identified as $\mathrm{CP}$ is an impairment of voluntary motor control. CP frequently involves one or more limbs and the trunk musculature and is classified by the type of motor abnormality (spasticity, athetosis, ataxia), area of the body that is affected (monoplegia, diplegia, hemiplegia, triplegia, tetraplegia) and location of lesion in the brain (pyramidal, extra pyramidal, mixed).Many studies have been conducted on rehab for CP children. But no study so far conducted has compared the effectiveness of supervised and non supervised home exercise program. Hence, the study was undertaken. On adherence to supervised and unsupervised home exercise program on motor milestones for spastic cerebral palsy.
\end{abstract}

Methods: 30 subjects diagnosed with spastic CP (3 months) were selected months.They were divided into two groups and treated with exercises for 2 months. Pre and post intervention outcomes were measured using GMFM(88) scale.

Results: Both the groups should improvement but there was significant on GMFM(88) scale in supervised group treated with exercises.

Conclusion: Home exercise program is effective in improving the motor milestones in Spastic CP patients.

Keywords: Spastic cerebral palsy; Supervised and unsupervised home exercise program; GMFM(88) scale

\section{Introduction}

Cerebral palsy is a sensory motor disorder that affects the control of posture and movement [1]. It is a common pediatric disorder occurring in about 2 to 2.5 per 1000 live births. It results from a non-progressive (static) insult to the developing brain. The child with CP is best cared for with an individualized treatment plan that provides a combination of interventions [2]. This requires the provision of a number of family-centered services that make a difference in the lives of these children and their families. Management of spasticity can be challenging with a wide variety of possible therapeutic interventions. The treatment must be goal oriented, such as to assist with mobility, reduce or prevent contractures, improve positioning and hygiene, and provide comfort [3]. Each member of the child's multidisciplinary team, including the child and both parents, should participate in the serial evaluations and treatment planning [4].

Cerebral palsy (CP) describes a group of developmental disorders of movement and posture, causing activity restriction or disability that is attributed to disturbances occurring in the fetal or infant brain. The motor impairments may be accompanied by a seizure disorder and by impairment of sensation, cognition, communication and behavior [5].

The hallmark sign of group of disorders identified as CP is an impairment of voluntary motor control. CP frequently involves one or more limbs and the trunk musculature and is classified by the type of motor abnormality (spasticity, athetosis, ataxia), area of the body that is affected (monoplegia, diplegia, hemiplegia, triplegia, tetraplegia) and location of lesion in the brain (pyramidal, extra pyramidal, mixed) [3]. Many studies have been conducted on rehab for CP children. But no study so far conducted has compared the effectiveness of supervised and non supervised home exercise program. Hence, the study was undertaken on adherence to supervised and unsupervised home exercise program on motor milestones for spastic cerebral palsy [6].

\section{Procedure}

Subject fulfilling the inclusion criteria were given consent form before intervention and explained regarding the study. 
Each subject had undergone the assessment for pre test values. Supervised group was taught a set of exercise program and asked to follow it for two months. During this two month intervention the supervised group was being observed by the therapist in the form of surprise visits, phone calls to the relatives, calling the patients family members and asking whether exercises were performed or not. Unsupervised group had been taught once the exercises and asked to follow it at home for two months. The exercises were performed twice a day for all week days for two months and 10 repetitions for each exercise each session was informed. Subjects were called for follow up and post test values were taken for supervised and unsupervised group.

\section{Intervention}

In the home exercise programme exercises were included according to the normal developmental milestones that is from neck control to gait training. A generalized protocol was set for both the groups and the exercises were chosen according to the patients need. The group of supervised patients was taught the exercises in group as all the patients of this group belong to one place and the unsupervised group patients some were taught individually and some in group. The supervised group was monitored by surprise visits and phone calls. The unsupervised group performed the same exercises without any supervision. The data was analyzed using GMFM(88) scale pre and post intervention.

\section{Statistical Analysis}

The Graph 1 shows comparison of mean values and standard deviation of dimension A of gross motor functional measure scale. The values were compared by applying paired $t$ test within the groups and unpaired t test in between two groups. Pre and post treatment values in supervised group show significant difference where $t=2.478, p=0.026$. In unsupervised group the pre and post treatment values show significant difference where $t=9.548, p=0.0001$. The post treatment values of both supervised and unsupervised group show very significant difference where $\mathrm{t}=2.26$ and $\mathrm{p}=0.03$.

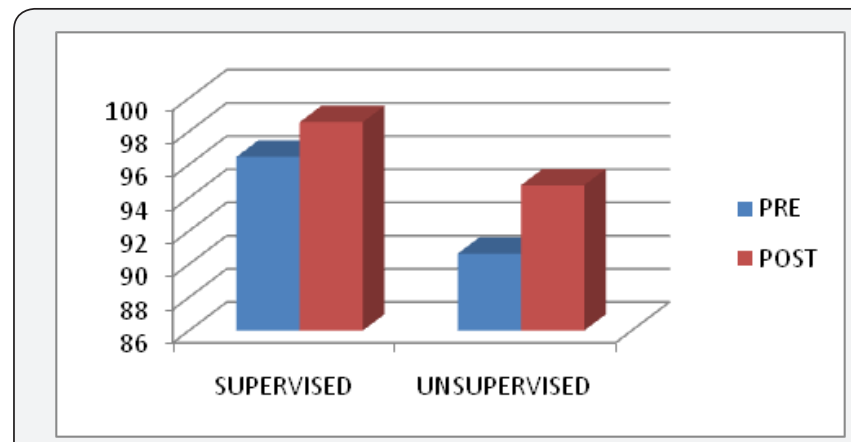

Graph 1: Comparison of mean values and standard deviation of dimension A of gross motor functional measure scale.

The Graph 2 shows comparison of mean values and standard deviation of dimension $\mathrm{B}$ of gross motor functional measure scale. The values were compared by applying paired $t$ test within the groups and unpaired t test in between two groups. Pre and treatment values in supervised group show extremely significant difference where $t=8.57, p=0.0001$. In unsupervised group the pre and post treatment values show extremely significant difference where $t=9.023, p=0.0001$. The post treatment values of both supervised and unsupervised group show very significant difference where $t=2.63$ and $p=0.01$.

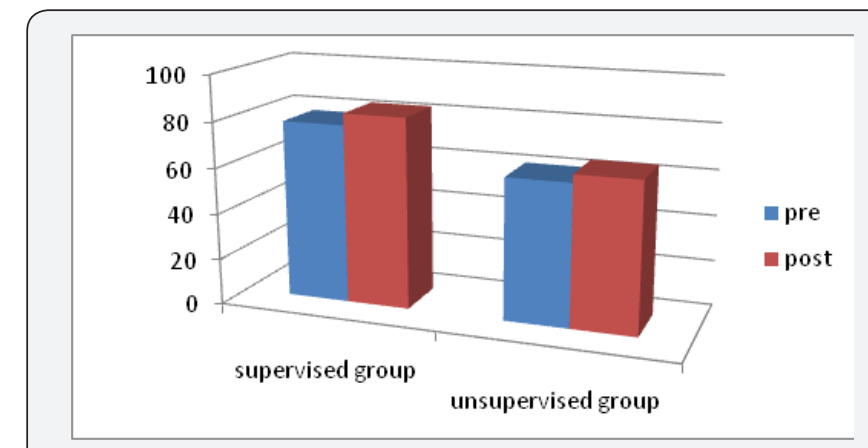

Graph 2: Comparison of mean values and standard deviation of dimension B of gross motor functional measure scale.

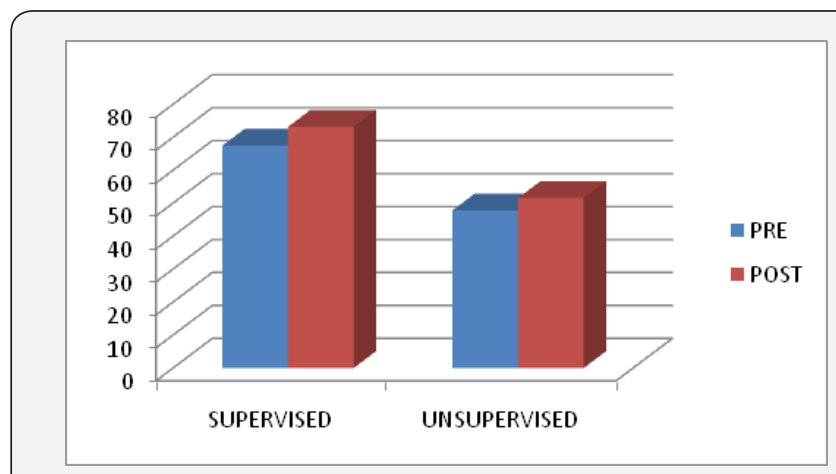

Graph 3: Comparison of mean values and standard deviation of dimension $\mathrm{C}$ of gross motor functional measure scale.

The Graph 3 shows comparison of mean values and standard deviation of dimension $\mathrm{C}$ of gross motor functional measure scale. The values were compared by applying paired t test within the groups and unpaired test in between two groups. Pre and treatment values in supervised group show very significant difference where $t=4.11, p=0.001$. In unsupervised group the pre and post treatment values show very significant difference where $t=5.61, p=0.0001$. The post treatment values of both supervised and unsupervised group show significant difference where $\mathrm{t}=2.19$ and $\mathrm{p}=0.03$.

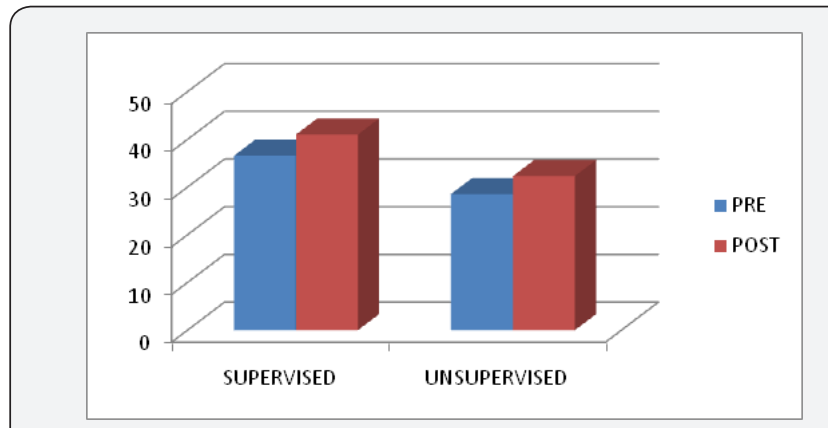

Graph 4: Comparison of mean values and standard deviation of dimension $\mathrm{D}$ of gross motor functional measure scale. 
The Graph 4 shows comparison of mean values and standard deviation of dimension D of gross motor functional measure scale. The values were compared by applying paired t test within the groups and unpaired t test in between two groups. Pre and treatment values in supervised group show very significant difference where $t=4.84, p=0.0003$. In unsupervised group the pre and post treatment values show very significant difference where $t=5.73, p=0.0001$. The post treatment values of both supervised and unsupervised group do not show significant difference where $\mathrm{t}=0.86$ and $\mathrm{p}=0.39$.

The Graph 5 shows comparison of mean values and standard deviation of dimension $\mathrm{E}$ of gross motor functional measure scale. The values were compared by applying paired t test within the groups and unpaired t test in between two groups. Pre and treatment values in supervised group show very significant difference where $t=3.14, p=0.007$. In unsupervised group the pre and post treatment values do not show significant difference where $\mathrm{t}=1.74, \mathrm{p}=0.1$. The post treatment values of both supervised and unsupervised group do not show significant difference where $\mathrm{t}=1.64$ and $\mathrm{p}=0.11$.

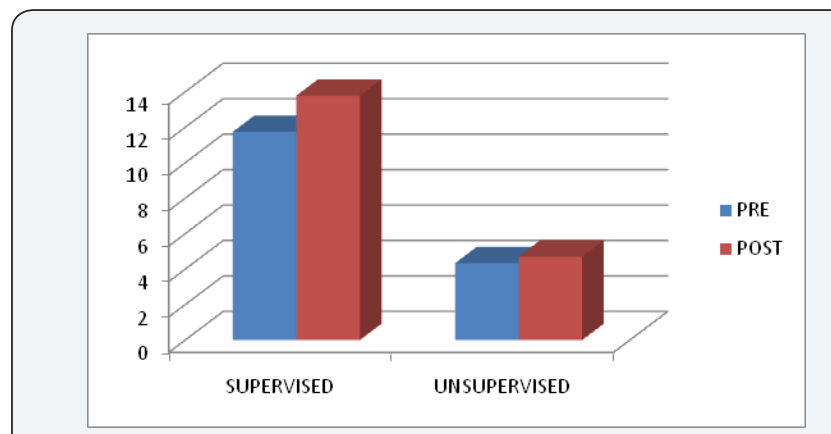

Graph 5: Comparison of mean values and standard deviation of dimension $\mathrm{E}$ of gross motor functional measure scale.

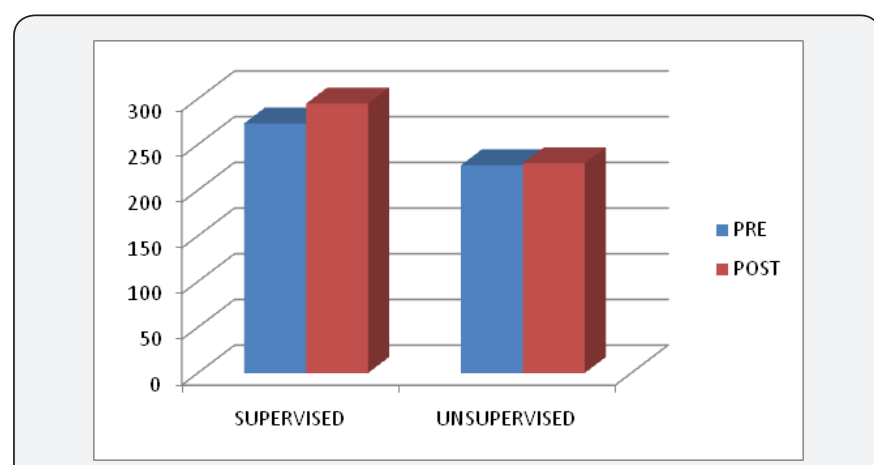

Graph 6: Comparison of mean values and standard deviation of TS of gross motor functional measure scale.

The Graph 6 shows comparison of mean values and standard deviation of TS of gross motor functional measure scale. The values were compared by applying paired t test within the groups and unpaired test in between two groups. Pre and treatment values in supervised group show very significant difference where $\mathrm{t}=4.24, \mathrm{p}=0.0008$. In unsupervised group the pre and post treatment values do not show significant difference where $\mathrm{t}=0.11, \mathrm{p}=0.91$. The post treatment values of both supervised and unsupervised group show significant difference where $\mathrm{t}=1.09$ and $\mathrm{p}=0.04$.

The Graph 7 shows comparison of mean values and standard deviation of GTS of gross motor functional measure scale. The values were compared by applying paired t test within the groups and unpaired test in between two groups. Pre and treatment values in supervised group show extremely significant difference where $t=4.64, p=0.0004$. In unsupervised group the pre and post treatment values show extremely significant difference where $\mathrm{t}=12.31, \mathrm{p}=0.0001$. The post treatment values of both supervised and unsupervised group show extremely significant difference where $\mathrm{t}=3.86$ and $\mathrm{p}=0.0006$.

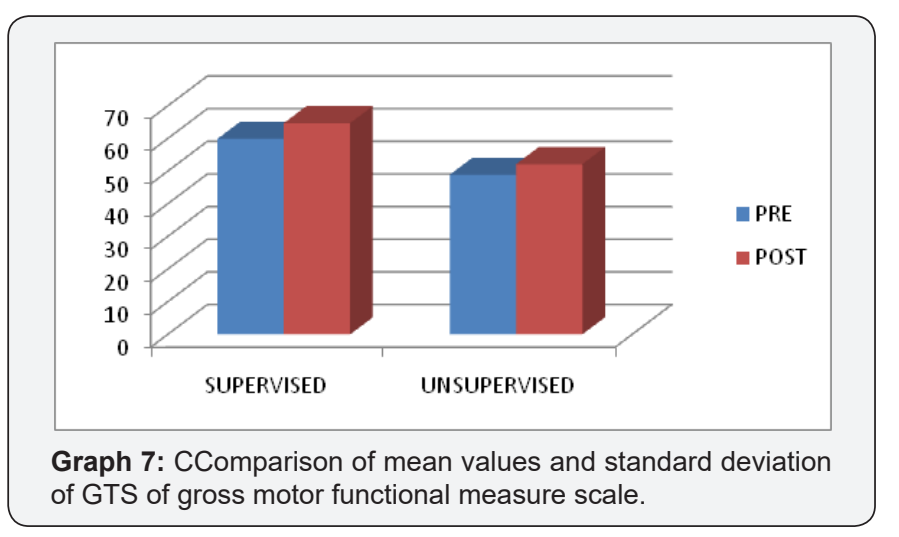

\section{Discussion}

Cerebral palsy (CP) is a common pediatric disorder occurring in about 2 to 2.5 per 1000 live births. It is a chronic motor disorder resulting from a non-progressive (static) insult to the developing brain. The research was conducted with the aim to study the adherence to supervised and unsupervised home exercise programme on motor milestones for spastic CP. 13 participants with age group of 3-14 years were included in the study and divided into supervised and unsupervised groups, where minimum age was 3.5 years and maximum 14 years for supervised group whereas minimum 3 years and maximum 12 years for unsupervised group. The supervised group was supervised by surprise visits and phone calls. The study included 18 males and 12 females. The conclusion was drawn by the outcome measures which were improvement in their adherence percentage and GMFM scale. Outcome measures in the study are adherence percentage and GMFM scale [7].

The motor milestones were assessed by using GMFM scale before treatment and on last day of treatment. The adherence percentage was calculated in combination with the GMFM scale. The GMFM is criterion-based observational measure. Content: The test includes 88 items that assess motor function in 5 dimensions: 1) lying and rolling; 2) sitting; 3) crawling and kneeling; 4) standing; and 5) walking, running, jumping. Because the aim of treatment was to maximize the child's potential for independent function, it was considered important to determine whether a child could complete the task independently (with 
or without the use of aids), without any active assistance from another person.

\section{Conclusion}

Based on the statistical results and interpretation it was concluded that the home exercise programme is effective in improving the motor milestones in spastic CP patients. Both the groups showed significant results post intervention but the supervised group showed more significant than the unsupervised group. Hence the alternate hypothesis was accepted.

\section{References}

1. Cook AS, Woollacott MH (2001) Motor control: theory and practical applications, $\left(2^{\text {nd }}\right.$ edn).
2. Jan MM (2006) Cerebral palsy: Comprehensive review and update. Ann Saudi Med 26(2): 123-132.

3. Downie PA, Cash JE (1986) Cash's textbook of neurology for physiotherapists, $\left(4^{\text {th }}\right.$ edn), pp. 517-520.

4. Campbell SK, Vander Linden DW, Palisano RJ (2006) Physical therapy for children, ( $4^{\text {th }}$ edn).

5. Tecklin JS (2008) Pediatric physical therapy, ( $4^{\text {th }}$ edn).

6. Mockford M, Caulton JM (2008) Systematic review of progressive strength training in children and adolescents with cerebral palsy who are ambulatory. Pediatr Phys Ther 20(4): 318-333.

7. Tang MH, Lin CK, Lin WH, Chen CH, Tsai SW, et al. (2011) The effect of adding a home program to weekly institutional-based therapy for children with undefined developmental delay: A pilot randomized clinical trial. J Chin Med Assoc 74(6): 259-266.

\section{Your next submission with Juniper Publishers} will reach you the below assets

- Quality Editorial service

- Swift Peer Review

- Reprints availability

- E-prints Service

- Manuscript Podcast for convenient understanding

- Global attainment for your research

- Manuscript accessibility in different formats ( Pdf, E-pub, Full Text, Audio)

- Unceasing customer service

Track the below URL for one-step submission https://juniperpublishers.com/online-submission.php 